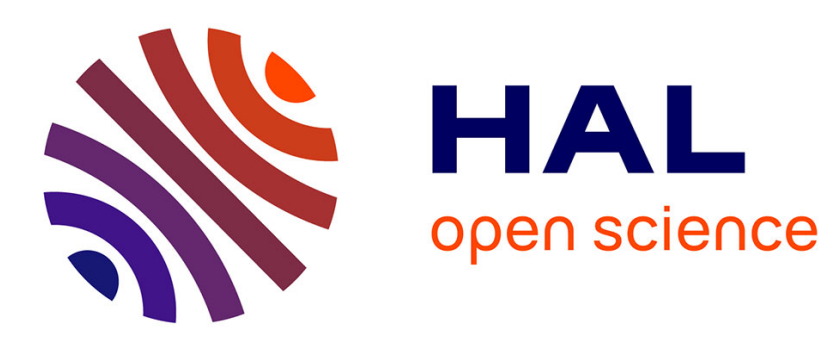

\title{
Les barrages face à la problématique des bois flottants: collecte, traitement et valorisation
}

Yves-François Le Lay, Bertrand Moulin

\section{To cite this version:}

Yves-François Le Lay, Bertrand Moulin. Les barrages face à la problématique des bois flottants: collecte, traitement et valorisation. La Houille Blanche - Revue internationale de l'eau, 2007, 3, pp.96-103. 10.1051/lhb:2007041 . halshs-00366449

\section{HAL Id: halshs-00366449 \\ https://shs.hal.science/halshs-00366449}

Submitted on 7 Mar 2009

HAL is a multi-disciplinary open access archive for the deposit and dissemination of scientific research documents, whether they are published or not. The documents may come from teaching and research institutions in France or abroad, or from public or private research centers.
L'archive ouverte pluridisciplinaire HAL, est destinée au dépôt et à la diffusion de documents scientifiques de niveau recherche, publiés ou non, émanant des établissements d'enseignement et de recherche français ou étrangers, des laboratoires publics ou privés. 
Le Lay Y.-F. ${ }^{1}$ et Moulin B. ${ }^{2}$, 2007. "Les barrages face à la problématique des bois flottants : collecte, traitement et valorisation". La Houille Blanche. Revue internationale de l'eau, 3, p. 96103.

${ }^{1}$ Université de Lyon, UMR 5600 "Environnement, Ville, Société", 18 rue Chevreul, F-69362

LYON cedex 07 ; e-mail : yf_lelay@hotmail.com

${ }^{2}$ CRENAM Université Jean Monnet - Saint-Etienne

\title{
Title
}

Dams and the issue of floating woods: gathering, treating and recovering

\section{Résumé}

Bien que les utilisateurs de prises d'eau ne soient pas responsables des entrées de bois dans les hydrosystèmes fluviaux, l'évolution de la législation et la demande sociale d'une protection accrue de l'environnement poussent les exploitants à reconsidérer leur gestion des corps flottants qui s'accumulent contre les barrages. Longtemps perçus comme une nuisance susceptible d'occasionner des pertes de charge, les bois flottants peuvent être valorisés, notamment dans le cadre du compostage et du chauffage. Mais cette valorisation reste tributaire de son insertion dans une filière dont le succès repose sur l'implication des différents acteurs à l'œuvre dans les territoires locaux de l'eau.

\begin{abstract}
Development companies are not responsible for the supply of large woody debris in fluvial hydrosystems. Nevertheless, the evolution of law and the social demand for an increased environmental conservation encourage decision-makers to reconsider their management of wastes accumulating against dams and hydraulic structures. Floating woods are not only a nuisance likely to induce economic costs, but also resources whose recovering is possible for the purposes of the composting and heating. The success depends on wood integration into a process of gathering, treating and recovering and on the capacity of all the stakeholders to involve in the maintenance of water territories.
\end{abstract}

\section{INTRODUCTION}

Les produits solides qui flottent à la surface des eaux (ou entre deux eaux) sont à l'origine de divers risques et nuisances [Le Lay, 2005 ; Le Lay et Piégay, 2007]. Leur présence le long des cours d'eau, au bord des retenues ou sur les plages du littoral dégrade le paysage [Le Lay et al., 2006]. Elle peut également provoquer des impacts sanitaires ou éco-toxicologiques, diminuer la qualité de l'eau, entraver l'écoulement et perturber l'exercice d'une activité économique [SMEAG / EPTB Garonne, 2004 ; Piégay et al., 2005]. Les déchets flottants posent un problème au fonctionnement en continu des organes ou équipements des barrages. Puisqu'ils sont susceptibles d'endommager les turbines des centrales hydroélectriques, des grilles de protection ont été mises en place aux prises d'eau. Mais les déchets s'accumulent contre celles-ci, les obstruent progressivement et provoquent des pertes de charges qui ont un coût économique pour l'usinier. Ce dernier doit éviter le colmatage des grilles et garantir le fonctionnement des turbines nécessaires à la production d'énergie hydroélectrique.

Les déchets flottants peuvent provenir des décharges, des campings et haltes nautiques, des exploitations agricoles, des industriels et artisans, ou encore des berges. Le bois y est toujours majoritaire et, si les exploitations forestières et les scieries produisent des déchets ligneux, le matériel constitutif des embâcles provient essentiellement de phénomènes naturels, en particulier de l'érosion des berges [Moulin et Piégay, 2004]. Les déchets flottants sont composés à $90 \%$ de bois sur le Rhin [E.D.F., 2002], à $80 \%$ sur le barrage de Saint-Egrève en Isère (communication 
personnelle des exploitants). Dans le Lot, l'expérimentation menée sur le barrage de Luzech [E.D.F., 1995a] a souligné la part considérable des débris végétaux dans les déchets collectés : bois (65\%), feuilles et fleurs (34 \%), et déchets assimilables aux ordures ménagères (1\%). Les gros bois (grosses branches, troncs et souches) posent les problèmes de gestion les plus importants : ils représentent $22 \%$ du volume total de déchets flottants à Saint-Egrève, entre 15 et $20 \%$ sur le Lot. Pour le bassin de l'Adour, la part des bois, fleurs et feuilles correspond à une fourchette de 80 à $99 \%$, le reste étant assimilable aux ordures ménagères [Institution Interdépartementale pour l'Aménagement Hydraulique du bassin de l'Adour, 1998]. En fait, il semble que la proportion de ces dernières varient selon que le site échantillonné se trouve à l'aval d'une zone très urbanisée et industrialisée (barrage de Saint-Egrève) ou peu urbanisée et industrialisée (barrage de Luzech).

Pendant longtemps, la gestion des déchets fut expéditive. Les solutions peu consommatrices en temps, en énergie et en argent étaient privilégiées. Il s'agissait d'en récupérer le moins possible... Deux procédés simples sont encore utilisés. La présence de prégrilles à l'entrée des canaux d'amenée permet d'orienter directement une part importante des déchets vers l'aval. D'autre part, les chasses permettent d'évacuer les corps flottants qui s'accumulent contre les grilles. En général, avant la chasse, les groupes sont arrêtés, ce qui produit un coup de bélier sur les grilles de prises d'eau. Les déchets s'en écartent. Dans le même temps, le clapet extérieur est abaissé ou la vanne ouverte : les déchets flottants sont entraînés par le courant à l'aval. Le procédé ne nécessite pas d'intervention humaine dans le cas des barrages déversants. Faute de moyens efficaces de récupération et de filières de traitement, nombreux sont ceux qui rejettent les volumes collectés en aval des installations... Mais les dossiers de renouvellement de concession ou d'autorisation offrent aux préfectures l'occasion d'intégrer dans les règlements d'eau des dispositions de récupération, de collecte et de traitement des déchets flottants.

Depuis la loi sur les déchets du 13 juillet 1992 (relative à l'élimination des déchets ainsi qu'aux installations classées pour la protection de l'environnement), l'exploitant devient le "propriétaire" des déchets sortis de l'eau (Encadré 1). Ces derniers ne sont pas considérés comme des déchets ultimes, c'est-à-dire non revalorisables, mais appartiennent plutôt à la catégorie des déchets industriels banals (D.I.B.). Il s'agit de "déchets assimilables aux ordures ménagères". Ils en contiennent en effet les mêmes composants (mais dans des proportions différentes) et les exploitants doivent supporter la charge de leur traitement [E.D.F., 1995b]. Pendant la seconde moitié des années 1990 et au début des années 2000, des études furent lancées car ces déchets ne devaient plus être acceptés en centre d'enfouissement technique après 2002 (en application de la loi du 13 juillet 1992). D'ailleurs, dans certains départements, l'administration soumet les renouvellements d'autorisation des microcentrales à l'acceptation d'une clause de récupération et d'élimination ou valorisation des déchets [Ministère de l'Ecologie et du Développement Durable, 2002]. Cet article montre comment des entreprises, au premier rang desquelles se place E.D.F., ont pris à leur compte le concept de développement durable pour que leur image bénéficie des retombées positives de leur prise en charge du nettoyage des cours d'eau et du traitement des déchets: le travail en partenariat dans le cadre d'une filière de valorisation, la participation d'entreprises d'insertion locales, la contribution à la protection de l'environnement et le développement des activités récréatives liées aux cours d'eau. Le traitement des bois flottants est plus particulièrement présenté dans quatre espaces structurés par l'Adour, l'Isère, le Rhin et le Rhône, soit des cours d'eau à fort débit.

Encadré 1 : Les déchets flottants dans le dispositif législatif français

La récupération des déchets flottants par les utilisateurs des prises d'eau relève d'un dispositif législatif qui a progressivement été élaboré sur le mode de la stratification :

- des lois sur les déchets. La loi n $75-633$ du 16 juillet 1975 relative à l'élimination des déchets et à la récupération des matériaux donnait cette définition générale à la notion de déchet: "(...) tout résidu d'un processus de production, de transformation ou d'utilisation, toute substance, matériau, produit ou plus généralement tout bien meuble abandonné ou que son détenteur destine à l'abandon" (Art. 1 ${ }^{\mathrm{er}}$ ). De plus, la loi n 92-646 du 13 juillet 1992 relative à l'élimination des déchets 
ainsi qu'aux installations classées pour la protection de l'environnement confère au producteur ou au détenteur du déchet l'obligation d'en assurer ou d'en faire assurer l'élimination dans des conditions propres à éviter les effets préjudiciables à l'environnement.

- des lois sur l'eau du 16 décembre 1964, du 3 janvier 1992 et du 30 décembre 2006 qui mentionnent l'interdiction de déverser des déchets susceptibles de porter atteinte à la santé publique et à l'environnement.

- du Code général des collectivités territoriales : "L'obligation générale d'entretien à laquelle sont soumis les propriétaires et affectataires du domaine public comporte celle d'éliminer ou de faire éliminer les déchets qui s'y trouvent" (Art. L2224-17).

\section{LA COLLECTE DES DECHETS FLOTTANTS}

\section{II.1 La récupération des déchets}

Deux types de sites sont particulièrement propices à la récupération des déchets flottants : les zones d'accumulations naturelles et les barrages. Peu d'études présentent la récupération sur les sites naturels (seuils, atterrissements, auxquels les ouvrages routiers peuvent être ajoutés). Elle est généralement organisée à la suite d'épisodes de crue, n'est pas automatisée et demande des moyens techniques mobiles pour intervenir depuis les berges ou sur l'eau. Cependant, la collecte en rivière peut être menée à l'aide de filets ou de divers pièges à flottants. Elle ne doit pas gêner les autres usages, notamment la navigation et les activités récréatives, et ne peut donc concerner que certains tronçons de cours d'eau. Sur la Nivelle (Pyrénées-Atlantiques), un filet fut installé avec succès sur un coude de la rivière, à l'aval du pont de l'A63, là où se formait une accumulation spontanée de déchets flottants. Semblable au filet utilisé par les pêcheurs, il s'étend sur 37 mètres de longueur et 1,10 mètres de hauteur ( $80 \mathrm{~cm}$ sous l'eau et $30 \mathrm{~cm}$ hors de l'eau). Pourvu d'une maille de $5 \mathrm{~cm}$, fixé à un point de mouillage de $300 \mathrm{~kg}$ ainsi qu'au perré par deux anneaux pris dans une glissière métallique, le dispositif permet d'accompagner la fluctuation des marées. Les $500 \mathrm{~m}^{3}$ de déchets piégés chaque année sont collectés par une association d'insertion (A.D.E.L.I.), puis amenés dans une décharge.

Les écluses et surtout les barrages hydroélectriques sont adaptés à une collecte régulière des déchets flottants. Mieux documentés, trois types de récupération sont mis en oeuvre : la drôme, le dégrillage et le bateau.

\section{II.1.1 Les drômes}

La collecte peut s'effectuer au moyen d'une drôme, c'est-à-dire un filet qui dévie les corps flottants vers un déversoir, une vanne ou une nappe d'accumulation. Installé dans les zones à faible courant, ce dispositif n'entrave pas complètement le transit des bois. Il est utilisé soit pour faciliter la récupération des flottants, soit pour favoriser leur évacuation à l'aval d'une infrastructure sensible. Mais les corps flottants ont tendance à se bloquer au niveau des ancrages ou à la jonction des éléments constitutifs du dispositif dont l'efficacité durable implique ainsi une surveillance et un entretien régulier.

\section{II.1.2 Le dégrillage}

Le dégrillage correspond à l'enlèvement régulier des corps flottants qui s'accumulent au niveau des grilles des prises d'eau. Sur de nombreux barrages, un dégrilleur est actionné en fonction des indications du détecteur de perte de charge aux grilles (Figure 1). La poche du dégrilleur descend le long de ces dernières et les nettoie : en fin de course, elle se referme sur les déchets, récupère ceux de petite et moyenne taille selon ses dimensions et remonte. Une fois en position haute, la poche s'ouvre et déverse les produits du dégrillage dans une goulotte d'évacuation ou sur une bande transporteuse. Dans le cas de la goulotte, l'eau qui y circule conduit les déchets vers l'aval et les déverse dans une fosse d'essorage ou une benne équipée d'un système d'évacuation de l'eau.

Lorsque la nappe de déchets flottants est trop épaisse pour que le dégrilleur puisse s'enfoncer sous le niveau de l'eau ou lorsque le diamètre, la longueur ou le poids des branches est trop grand pour 
qu'il parvienne à saisir les éléments, la décision d'effectuer une chasse est prise et un dégrillage d'affinage peut être ensuite réalisé pour collecter les feuilles et les plastiques restés collés aux grilles. Néanmoins, l'adjonction d'un grappin permet de saisir les branchages coincés dans les grillages et susceptibles de bloquer le dégrilleur, ainsi que les troncs et les souches que ce dernier ne peut remonter en raison de leur trop grandes dimensions. Les matériaux sont déposés sur une plateforme où ils sont débités avant d'être déposés dans une goulotte ou utilisés pour la consommation personnelle de particuliers.

De plus, dans tous les cas, seule une partie des déchets est récupérable. En période de crue, la collecte peut s'avérer difficile. Le débit dirigé vers la prise d'eau ne peut qu'être inférieur ou égal au débit maximum turbinable. L'utilisation des chasses évacue alors également les débris ligneux.

\section{II.1.3 La barge}

Certains barrages ne disposent pas de dégrilleur. Les exploitants recourent alors aux bateaux, comme sur la retenue de Grangent dans la Loire et celle de Génissiat sur le Rhône. La barge évolue sur le plan d'eau, devant les grilles, et récupère les corps flottants de dimensions moyennes et grandes à l'aide d'un grappin. Au fur et à mesure, les matériaux non biodégradables sont triés et stockés dans un petit container. La barge peut ensuite décharger ses déchets dans différentes bennes de stockage, par exemple une grande pour les bois et les feuilles et une plus petite pour les autres déchets (ménagers, etc...). Le bateau peut être muni, à l'avant, d'un tapis roulant grillagé et métallique qui plonge dans l'eau et ramasse progressivement les corps flottants. Leur transit sur la bande transporteuse facilite la séparation des matériaux. D'une façon semblable, le Port autonome de Paris utilisent des bateaux nettoyeur qui interviennent notamment sur la Seine parisienne. L'inconvénient de la barge réside en ceci qu'elle ne peut être utilisée que pour des débits modérés et des corps flottants de petite taille.

Le barrage de Génissiat, le plus grand du Haut Rhône français, bloque intégralement l'écoulement et le transit des corps flottants. Dépourvu de dégrilleur, le bois s'accumulait contre le barrage d'autant plus rapidement que la gestion suisse reposait sur la pratique des chasses jusqu'en 2001. Pour éviter que les bois coulent, leur extraction est systématique depuis 1989, trois à quatre fois par an [Moulin, 1999]. Le procédé est très contraignant : l'extraction de $5000 \mathrm{~m}^{3}$ dure une dizaine de jours et coûte plusieurs dizaines de milliers d'euros. Un bateau pousseur regroupe les corps flottants et pousse la nappe en direction de l'aire d'extraction, en rive droite (Figure 2). Une grue extrait les bois et charge des camions-bennes qui se dirigent ensuite vers une plate-forme de stockage Un tri élémentaire est réalisé en distinguant les bennes de bois des autres déchets.

\section{II.2 Le tri des déchets flottants}

La valorisation d'une partie des déchets récupérés sur les ouvrages hydrauliques nécessite un tri préalable avant traitement. Cette étape est contraignante en raison de l'hétérogénéité des déchets flottants et du travail peu gratifiant qu'elle demande. Il s'agit de séparer la portion valorisable des résidus destinés à l'élimination.

Plus le tri est précoce, plus il est efficace. S'il est effectué dans les bennes, l'enchevêtrement des matériaux le complique. Et les petits éléments en plastique, les boulettes de polystyrène, les métaux ou le verre brisé sont d'accès difficile sous les troncs et branchages... Or, des matériaux mal triés sont plus difficilement valorisables et n'intéressent pas les éventuels repreneurs. En fait, une fois dans la goulotte, les matériaux sont très enchevêtrés et le verre est cassé. Le tri est déjà compliqué... C'est pourquoi l'usage d'une bande transporteuse poreuse est utile. L'enlèvement des matériaux non biodégradables y est possible et l'égouttage des déchets sur le trajet permet leur stockage dans une benne classique. Cependant, la dangerosité des bouteilles de verre cassées et le nombre et la petite taille des boulettes de polystyrène continuent de faire problème.

Deux approches principales existent (Tableau 1) :

- la séparation du bois (barrage de Saint-Egrève). Un tri sommaire est effectué pour isoler mécaniquement les gros déchets indésirables et les métaux. Il n'y a pas de tri manuel. 
- la séparation multiflux (barrage d'Artix). Un tri manuel complémentaire est obligatoire pour séparer les différents produits, à savoir les bois, les déchets recyclables (verre, métaux, plastiques), les déchets toxiques et le tout-venant non valorisable.

Plus généralement, le broyage du bois issu du tri des déchets flottants reste une opération nécessaire (avant le compostage) et difficile. Les dimensions (longueur et diamètre) des gros bois et des souches doivent être préalablement réduites. Or, le débitage de ces dernières en morceaux plus petits est malaisé et leur réseau racinaire comporte des cailloux. Le matériel subit une usure prématurée parce que :

- le produit à broyer contient fréquemment du sable ou des pierres ;

- le bois peut être très dur lorsqu'il a longtemps séjourné dans l'eau ;

- des essences différentes sont présentes.

\section{LE TRAITEMENT DES BOIS : ELIMINATION ET VALORISATION}

Une fois les déchets flottants extraits, ils doivent être traités (Figure 3). Le bois restant une matière et une source d'énergie, sa valorisation (compostage et bois de chauffe) se développe aux dépens de son élimination (enfouissement et incinération). Malgré des initiatives innovantes, il trouve cependant inégalement sa place dans les filières locales de traitement (Tableau 2).

\section{III.1 L'élimination des bois flottants}

\section{III.1.1 L'enfouissement}

Théoriquement, les bois flottants peuvent être éliminés par stockage ou par brûlage. Les décharges traditionnelles ayant disparu progressivement, le Centre d'Enfouissement Technique peut apparaître comme une solution. Mais, depuis juillet 2002, les installations d'élimination des déchets par stockage ne sont plus autorisées qu'à accueillir les déchets ultimes. La loi du 13 juillet 1992 en donnait une définition : "est ultime (...) un déchet résultant ou non du traitement d'un déchet, qui n'est plus susceptible d'être traité dans les conditions techniques et économiques du moment, notamment par extraction de la part valorisable ou par réduction de son caractère polluant ou dangereux". La circulaire du 28 avril 1998, relative à la mise en œuvre et à l'évolution des plans départementaux d'élimination des déchets ménagers et assimilés, a redéfini la notion. "Le déchet ultime (...) se définit comme la fraction non « récupérable » des déchets et non comme le seul résidu de l'incinération". Il procède de l'extraction préalable, par collecte sélective ou par tri, des matériaux (emballages, verres, papier, carton, plastique, journaux...), de la fraction biodégradable destinée à un traitement biologique (compostage et méthanisation) ou à l'épandage agricole, et des produits usagés qui doivent intégrer des filières spécifiques (huiles usées, piles et accumulateurs...). Le stockage des déchets flottants ne peut donc être envisagé qu'après l'extraction de la fraction valorisable et toxique. Le bois ne peut plus être enfoui.

\section{III.1.2 L'incinération}

Malgré des pratiques ponctuellement persistantes, le brûlage à l'air libre des déchets industriels banals est illégal. Qu'en est-il de l'incinération des bois flottants ? Une Unité d'Incinération d'Ordures Ménagères (U.I.O.M.) est conçue et dimensionnée pour brûler continuellement l'ensemble des déchets urbains pendant une vingtaine d'années au minimum. Selon qu'elle pratique ou non une valorisation énergétique, l'usine d'incinération est considérée comme une installation de valorisation ou d'élimination. Elle traite les ordures ménagères brutes et les refus de tri ; mais elle peut également recevoir les encombrants incinérables qui proviennent des déchetteries, ainsi que les déchets industriels banals. Les bois récupérés contre les barrages peuvent prétendre à cette dernière catégorie. L'incinération des déchets flottants nécessitent cependant des tours à combustion équipées de filtres. Elle implique également que les dimensions des bois soient limitées pour répondre aux caractéristiques du four et que le taux de sable soit faible. Tels quels, les gros bois et les souches en sont donc exclus. 


\section{III.2 La valorisation des bois flottants}

$\mathrm{Au}$ sein de la filière bois, plusieurs sous-produits proviennent de l'ensemble des travaux depuis l'exploitation forestière jusqu'au bois en fin de vie, en passant par les travaux de façonnage de la scierie à la fabrication de produits finis. Les "déchets de bois" sont définis selon leur provenance :

- les déchets de première transformation (écorces, sciures et chutes), dits déchets humides ;

- les déchets de seconde transformation (sciures, copeaux et chutes), dits déchets secs ;

- les bois de rebut (bois en fin de vie tels que les palettes, les cagettes ou les meubles).

En général, les déchets ligneux sont valorisés comme énergie ou comme matière. Cependant, le bois récupéré dans les cours d'eau n'est bien intégré ni dans le processus de fabrication de pâte à papier (qui nécessite des déchets humides), ni dans celui des panneaux de particules et des complexes bois/plastique ou bois/béton (pour lesquels la plupart des types de bois conviennent). Les usines utilisent principalement des essences résineuses et les mélanges d'essence ne sont pas toujours permis.

Restent donc le compostage et la valorisation énergétique. Mais des problèmes émergent à nouveau. En particulier, les bois flottants n'étant pas tous d'origine naturelle, certains ont été traités. Or la présence de vernis, de peinture ou de colle constitue une contrainte supplémentaire lors de la recherche d'une valorisation.

\section{III.2.1 Le compostage}

Le compostage des déchets à l'air libre consiste à dégrader la matière organique. Des processus microbiologiques aérobies la transforment en substances humides et minérales qui constituent des éléments fertilisants, du fait de la présence de nitrates et phosphates notamment. Néanmoins, la qualité et les débouchés potentiels du compost dépendent de la nature des déchets qui sont entrés dans sa composition. Les composts les plus recherchés sont issus de déchets verts et de fumiers. La diversité du contenu des déchets flottants confère au compost une image de moins bonne qualité aux yeux des utilisateurs potentiels.

La compostabilité des produits varie selon leur teneur en carbone $(\mathrm{C})$ et en azote $(\mathrm{N})$, l'humidité relative, leur acidité ou encore l'aération du milieu. Le faible rapport $\mathrm{C} / \mathrm{N}$ des feuilles, comme celui des tontes de gazon, n'est pas favorable au compostage : elles se dégradent rapidement, émettant une odeur putride... Les feuilles doivent être mélangées avec d'autres déchets dits "structuraux" qui créent des voies d'oxygénation et optimisent les échanges entre gaz et eau. Après leur broyage, les branches sont considérées comme des éléments structurants qui présentent l'intérêt d'être riches en lignine et l'inconvénient de se caractériser par une vitesse de biodégradabilité lente. Globalement, les déchets en mélange possèdent une bonne aptitude au compostage. Le traitement conjoint des bois, branches et feuilles issus du tri des déchets flottants avec les déchets verts constitue un véritable débouché. Il importe cependant de s'assurer que les déchets à composter sont démunis d'impuretés (plastiques, verre, métaux...) et de micro-organismes pathogènes (chancre du platane ou feu bactérien).

Le compostage connut un bref succès dans le cadre de la valorisation des bois flottants extraits au barrage de Génissiat. Les exploitants ont d'abord compté sur les particuliers pour écouler le stock constitué en rive droite. Mais cette invitation officieuse n'aboutit qu'à un faible prélèvement. Les responsables cherchèrent donc des filières de valorisation, démarchant notamment les papeteries jusqu'en Hollande. Cette piste demeura infructueuse en raison des caractéristiques insatisfaisantes de la matière première : dégradation du bois, géométrie plus complexe que les fûts habituels, diversité des essences... Seul le compostage constitua temporairement une solution sérieuse grâce à VEGERHONE, une société spécialisée dans le recyclage des déchets verts de la région lyonnaise. Elle est née de l'association de trois entreprises :

- la Compagnie Nationale du Rhône (C.N.R.) dont l'intérêt est de trouver une solution, compatible avec la réglementation, pour évacuer les bois flottants collectés sur les barrages ; 
- la Société Ouest Service Entreprise (O.S.E.) spécialisée dans le ramassage des déchets et chargée de la gestion de déchetteries dans la région lyonnaise ;

- la Société MICHUT spécialisée dans la fabrication et la commercialisation d'amendements organiques.

Le centre de broyage se situait au port Edouard Herriot. Un pont-bascule pesait précisément le chargement des camions. Dès leur réception, les déchets étaient stockés pour un tri manuel sommaire qui visait à mettre de côté les éléments indésirables (notamment les objets métalliques, plastiques et cailloux). Le broyeur fixe de VEGERHONE permettait le traitement de 60000 tonnes de déchets par an. Le compostage était effectué sur deux plateformes, l'une dans le Rhône et l'autre en Isère, à base de déchets végétaux (produits par le Grand Lyon et les particuliers) qui étaient collectés par les déchetteries. Mais, dans le cadre de la charte de l'environnement qui engageait la C.N.R. à mieux gérer le fleuve dont elle est concessionnaire, des campagnes de compostage furent conduites, au début des années 1990, avec les déchets flottants récupérés sur le site de Génissiat. Les bois étaient broyés au niveau du barrage (Figure 4); et la plateforme iséroise réceptionnait les broyats qui étaient ensuite mélangés avec d'autres déchets verts et mis en andains. Le retournement et l'aération des andains étaient effectués régulièrement pour améliorer le processus de dégradation du produit, l'homogénéiser et renouveler l'air. Après la mise en maturation, le criblage, l'élimination des refus de compostage et l'affinage de l'amendement, le produit fini était stocké en vue de sa commercialisation. Il servait d'amendement pour les cultures pérennes de la vallée du Rhône (viticulture, arboriculture, maraîchage et pépinières) et pour l'entretien des espaces verts. Il constituait également une base pour l'élaboration d'engrais et de composts spécifiques. Mais les débouchés s'étant avérés insuffisants, VEGERHONE n'existe plus. Aujourd'hui, le bois issu du barrage de Génissiat est broyé ; puis la sciure est transportée en Italie, où elle est notamment utilisée pour le chauffage. Un nouveau projet est actuellement à l'étude. Le remontage d'une structure, dépendante du groupe Suez-Electrabel, est envisagé pour traiter le bois extrait contre l'ensemble des barrages du Rhône. La valorisation déboucherait sur du bois de chauffe à destination de centrales thermiques.

\section{III.2.2 Le bois de chauffe}

Les sous-produits du bois sont valorisables en tant que combustibles (Tableau 3). Cette valorisation énergétique nécessite des chaudières à alimentation automatique en bois. Les entreprises de transformation du bois satisfont ainsi leur propre besoin en énergie. Des chaufferies collectives et industrielles fonctionnent également de la sorte. La principale contrainte réside dans la qualité du combustible. Ce dernier doit présenter une granulométrie homogène après broyage et avoir été suffisamment séché et correctement déferraillé. La valorisation des déchets humides exige des chaufferies de grosse puissance, utilisées principalement dans l'industrie ou pour les bâtiments à usages collectifs (par exemple des lycées ou des groupes de logements).

\section{VERS UNE GESTION INTEGREE DES DECHETS FLOTTANTS}

\section{IV.1 L'Adour : des déchets littoraux à une gestion par bassin}

Le Schéma Directeur d'Aménagement et de Gestion des Eaux (S.D.A.G.E.) Adour-Garonne, approuvé en 1996, reste le seul qui préconise une considération collective des déchets flottants. "Dans le cadre de schémas définis par cours d'eau ou bassin versant", sa mesure A17 recommande que "la nature et le volume de ces déchets soient pris en compte par les plans départementaux d'élimination des déchets ménagers et assimilés" et que "la récupération des déchets soit faite sur chaque ouvrage, ou sur certains d'entre eux par les exploitants de ces ouvrages".

Si la réflexion concerne aujourd'hui l'Agout et la Garonne [SMEAG / EPTB Garonne, 2004], l'Institution Interdépartementale pour l'Aménagement Hydraulique du bassin de l'Adour [1998 et 1999], regroupant quatre départements, fut le premier maître d'ouvrage à lancer "une démarche de planification cohérente de gestion des flottants", à l'interface des politiques de gestion des déchets et des politiques d'entretien et de restauration des cours d'eau et des plages du littoral [Darthou, 1998]. 
Cette approche associe une logique préventive, destinée à réduire la quantité des apports, et une logique curative dont l'objet est de collecter et traiter les déchets. Cette précocité s'explique par l'incident de la décharge de La Corogne (Espagne) dont les 200000 tonnes de déchets ménagers (et assimilés), glissés en mer en septembre 1996, ont aggravé la pollution du littoral basco-landais. Durant les étés 1997 et 1998, la quasi-totalité des plages aquitaines ont enregistré des échouages massifs et continus. Les gestionnaires estiment que les macrodéchets flottants dégradent le paysage et "rendent le milieu peu accueillant pour l'activité touristique" [Observatoire de l'Eau des Pays de l'Adour, 2000]. Les dépôts sont également source de nuisance olfactive et représentent une menace pour certaines espèces marines.

Le volume des corps flottants collectés sur les plages basques et landaises a été estimé à une fourchette qui s'étend entre 17900 et $37200 \mathrm{~m}^{3} / \mathrm{an}$ [Institution Interdépartementale pour l'Aménagement Hydraulique du bassin de l'Adour, 1998]. Ces macrodéchets sont composés des matériaux les plus divers :

- des bois et branchages issus, en partie, du bassin de l'Adour ;

- des déchets assimilables à des ordures ménagères (déchets alimentaires), avec un pourcentage important de plastiques (emballages) ;

- des déchets liés aux activités de pêche (filets et cordages) ou au rejet des navires ;

- des cadavres d'animaux (d'origine terrestre et marine).

Les bois constituent généralement le composant principal des déchets flottants échoués sur le littoral aquitain : entre 30 et $70 \%$ selon la saison et le secteur échantillonné.

La totalité des déchets est évacuée sélectivement. Les cadavres d'animaux sont pris en charge par un équarisseur. Des filières spécialisées collectent spécifiquement et détruisent les objets dangereux. Mais les déchets sont essentiellement constitués de bois. Les gros bois sont triés, broyés et conditionnés en plaquettes pour être incorporés dans la filière "bois-énergie" du Conseil Général des Landes qui dispose d'une quinzaine de chaufferies-bois dans le département. Le brûlage au sein de chaufferies doit respecter des conditions réglementaires. Celles-ci ne sont satisfaites qu'au moyen d'un produit exempt d'impuretés et présentant une granulométrie et une humidité homogènes. Des expérimentations de broyage fin du bois ont été conduites pour l'incorporer à des boues de stations d'épuration destinées à l'épandage. Quant aux autres déchets, mélangés et rassemblés dans des containers, ils sont ensuite acheminés vers des plateformes de tri où ils sont dirigés vers les usines d'incinération selon leur nature.

La diversité des phénomènes physiques qui aboutissent au dépôt de déchets sur les plages aquitaines ne permet pas d'évaluer avec précision quelle est la contribution des cours d'eau. Néanmoins, des mesures sont mises en oeuvre à l'amont de la problématique de gestion des déchets flottants sur le littoral. Une action de prévention - la "politique rivière" - a été développée par le Conseil Général des Pyrénées-Atlantiques, en partenariat avec l'Agence de l'Eau Adour-Garonne. A l'échelle des bassins versants, cette politique contractuelle se donne notamment pour priorité la réhabilitation et l'entretien des cours d'eau et des milieux aquatiques. Une gestion concertée doit favoriser l'émergence de maîtres d'ouvrage pour la réalisation et le suivi des travaux : il s'agit d'organiser l'entretien pérenne des principaux cours d'eau (nettoyage du lit et des berges, et gestion de la végétation stabilisatrice de ces dernières) dans le but de réduire les déchets flottants.

\section{IV.2 Le bois flottant dans la filière de recyclage du bois en Isère}

En Isère, les responsables publics et privés ont tôt pris conscience de la nécessité du recyclage (Figure 5). Sous l'impulsion de la D.R.I.R.E., un Comité pour l'Elimination Rationnelle des Déchets en Isère (C.O.P.E.R.D.I.) a été créé en 1992, en réunissant des représentants de l'Etat et du département, de l'Association des maires, des industriels producteurs et éliminateurs, des associations de protection de l'environnement et de consommateurs, et des organismes publics (A.D.E.M.E. et Agence de l'eau). Dans le cadre du schéma de traitement et d'élimination des déchets industriels banals, une filière de récupération du bois a été développée grâce au partenariat 
d'un producteur (E.D.F), d'un recycleur (LELY Evac'ordures) et d'un consommateur (la Compagnie de Chauffage de l'agglomération grenobloise).

Le barrage-usine de Saint-Egrève se situe à l'aval de Grenoble et de la confluence Drac-Isère. Mis en service en 1990, il fut construit avec un souci de bonne intégration dans l'agglomération grenobloise. Le barrage est équipé de cinq vannes et crée une chute de 11,50 mètres. Pourvue de deux groupes bulbes de $250 \mathrm{~m}^{3} / \mathrm{s}$, la centrale se caractérise par une puissance de $46 \mathrm{MW}$. Mais le barrage interrompt le transit des corps flottants sur l'Isère et des matériaux divers s'accumulent contre les grilles : de grosses quantités de bois, mais aussi des plastiques, des métaux, des aérosols, des bidons, des bouteilles de gaz, des pneus... La conception du système de récupération a été menée en même temps que celle du barrage, ce qui a permis d'optimiser le fonctionnement de l'installation, en service depuis le début de 1994. A ce titre, l'aménagement de Saint-Egrève est un exemple de ce qu'il est possible de faire en termes de récupération et de valorisation des déchets flottants.

Le dégrilleur se trouve à l'amont de l'usine. Les déchets enlevés sont ensuite acheminés au moyen d'un canal de défeuillage qui est alimenté par une vanne motorisée. 400 1/s sont nécessaires au portage des matériaux dégrillés; ils doivent être débités même lorsque la cote minimale d'exploitation est atteinte. Quant aux bois dont les dimensions sont trop importantes pour être dégrillés, ils sont récupérés à l'aide d'une grue mobile et équipée d'un grappin forestier. Entreposés sur une plateforme en rive droite, ils y sont ensuite découpés avant leur transport à la station de broyage.

Le canal de défeuillage chemine jusqu'à un aiguillage qui permet l'orientation soit vers la fosse d'essorage et la station de broyage, soit vers l'aval. Par flottaison, les déchets gagnent la fosse d'essorage de $240 \mathrm{~m}^{3}$. Un détecteur de métaux installé sur le canal d'amenée permet d'écarter à l'aide d'un clapet d'évacuation les objets métalliques, notamment les bouteilles de gaz susceptibles d'exploser lors du broyage. Une grue hydraulique équipée d'un grappin à cinq doigts reprend les déchets présents dans la fosse d'essorage pour les déposer dans la trémie du broyeur. Ce dernier peut absorber tous les types de déchets, à l'exception des bouteilles de gaz. A la sortie du broyeur, un transporteur à bandes récupère les déchets broyés et les déverse dans la trémie d'un second tapis de type rotatif qui assure le remplissage de quatre bennes disposées en étoile.

Les bennes gagnent la station de Saint-Quentin-en-Isère, opérationnelle depuis juin 1994, où LELY-Evac'ordures recycle le bois. L'entreprise poursuit le triage des déchets. Deux sortes de bennes sont distinguées. Les "bennes sales" contiennent trop de déchets non ligneux pour une valorisation, alors que le contenu des "bennes propres" peut être utilisé comme combustible. Les grosses pièces sont cassées à l'aide d'une déchiqueteuse mobile. Après un premier déferraillage, le bois est séché et stocké. Une fois réduit en plaquettes de $5 \mathrm{~cm}$ par un broyeur de grande capacité, il est totalement déferraillé. Le combustible sec qui en résulte peut alors être expédié aux utilisateurs. Des stocks de plusieurs mois sont néanmoins constitués puisque la période de production des plaquettes, de mars à septembre, ne coïncide pas avec la période de consommation, d'octobre à mars.

En particulier, 10000 tonnes de plaquettes de bonne qualité alimentent les chaudières de la Compagnie de Chauffage de l'agglomération grenobloise qui emploie également le charbon, le fuel, les ordures ménagères, l'électricité et le gaz. La centrale de Villeneuve est équipée pour une alimentation en déchets de bois. Grossièrement, deux tonnes de bois apportent l'équivalent calorique d'une tonne de charbon. Par ailleurs, la combustion du bois présente l'intérêt de ne pas dégager de soufre.

E.D.F. a donc externalisé le transport, le séchage, le broyage complémentaire, le stockage et la livraison. Mais, depuis la mise en service de l'installation, le taux de valorisation est passé de 74 à $30 \%$ des déchets récupérés. Les exigences concernant la qualité du bois sont de plus en plus strictes, si bien que les feuilles et les branchages ne sont plus valorisés. La possibilité d'un tri partiellement manuel sur le site est évoquée. 


\section{IV.3 L'émergence d'une valorisation des bois flottants sur le Rhin}

La masse de détritus extraite du Rhin au niveau des centrales E.D.F. varie entre 5000 et 15000 tonnes par an [E.D.F., 2002; Conseil National du Développement Durable, 2003]. L'ensemble représente en moyenne $7000 \mathrm{~m}^{3}$ de matériaux, dont $4500 \mathrm{~m}^{3}$ pour la seule centrale de Kembs. La récupération des dépôts est assurée par les exploitants des centrales. En période de crue - par exemple lorsque le débit du fleuve dépasse $2800 \mathrm{~m}^{3} / \mathrm{s}-$ des agents se relayent nuit et jour pour extraire les corps flottants.

L'enfouissement constituait une solution peu respectueuse de l'environnement et présentait également l'inconvénient d'être coûteuse : 110 euros par tonne de déchets traitée. Du fait de l'évolution de la législation et d'une volonté d'inscrire son activité dans le cadre du développement durable, EDF Unité de Production Est a cherché à trier et valoriser ses déchets extraits du cours d'eau. Elle confie la prestation du traitement des détritus à une entreprise d'insertion, AU PORT'Unes. Le tri manuel est effectué sur le site pour éviter un surcoût. Il permet de séparer les déchets industriels (plastiques, pneus, bidons...) des déchets végétaux. Concernant la valorisation de ces derniers, plusieurs possibilités ont été envisagées : le traitement en compost, la fabrication de briquettes et le bois de chauffage domestique.

Bien que l'équivalent de 25 à 75 hectares de forêt soit récupéré chaque année, le bois extrait ne se prête pas au chauffage domestique parce qu'il provient principalement d'arbres au bois tendre, tels que les saules et les peupliers. N'ayant pas identifié un partenaire intéressé pour le compostage, E.D.F. s'est donc tourné vers la production de briquettes. Rechignant à acquérir un broyeur capable de broyer les souches, ce qui implique un investissement de départ important et le traitement d'une grande quantité de bois, E.D.F. préfère louer les services d'un broyeur mobile. Puis, l'entreprise locale STRACEL prend en charge l'évacuation des déchets végétaux triés et s'en sert pour le chauffage. Fabricant de papier journal, elle achetait une grande quantité de bois de chauffe dépourvu de tout corps étranger (50 000 t/an) qu'elle remplace désormais en partie par des déchets végétaux.

Concernant en premier lieu la centrale hydroélectrique de Strasbourg, le projet a été élargi en 2002 aux centrales de Gerstheim et Gambsheim. Il a permis la création d'un poste "Développement/Environnement" dans l'entreprise locale. De plus, sur le site de Strasbourg, E.D.F. devrait abaisser le coût du traitement d'une tonne de détritus de 110,30 à 32,54 euros. Ramené à l'ensemble des centrales du Rhin, E.D.F. envisage une économie variant entre 388800 et 1166400 euros par an susceptible de faciliter un investissement dans le recyclage des autres déchets (déchets industriels banals et déchets industriels spéciaux). Le programme peut être étendu aux neuf sites sur le Rhin, en recourant au transport fluvial des déchets.

\section{CONCLUSION}

Bien que le bois remplisse un rôle important dans le fonctionnement des écosystèmes d'eau courante [Gregory et al., 2003], il constitue une nuisance pour les exploitants de barrages hydrauliques. La notion de déchets flottants est ambiguë au regard du dispositif législatif français. Certes, les exploitants de ces ouvrages ne sont pas responsables des entrées de bois dans les hydrosystèmes fluviaux. Mais l'évolution de la législation et la demande sociale d'une protection accrue de l'environnement - y compris celle des bribes de nature urbaine que peuvent constituer les cours d'eau aménagés - ont poussé les exploitants à reconsidérer leur gestion des bois flottants qui s'accumulent contre les barrages. Si le rejet des bois flottants à l'aval des installations est commode, il s'avère plus gratifiant de les valoriser. Quelques pistes ont été explorées, notamment dans le cadre du chauffage et du compostage, qui exigent toutes une phase préalable de collecte et de traitement. La valorisation des bois flottants est possible, mais reste tributaire de son insertion dans une filière dont le succès repose sur l'implication des décideurs, gestionnaires et acteurs à l'œuvre dans les différents territoires locaux de l'eau. 


\section{REMERCIEMENTS}

Les auteurs remercient chaleureusement Anne Rivière-Honegger pour ses conseils bienveillants et sa relecture attentive.

\section{VIIRÉFÉRENCES BIBLIOGRAPHIQUES}

Alexandre, O. \& Arrus, R. 2005. Les «territoires» de l'eau. http://www.cybergeo.presse.fr/eauville/arrus.htm.

Darthou, F. 1998. Politique pour une gestion des « déchets » flottants. La Houille Blanche, 8, 57-60.

E.D.F. 2002. Enlèvement et valorisation des déchets flottants du Rhin à la centrale hydroélectrique de Strasbourg. http://www.aufildurhin.com/fr/actualites/trophees2.pdf

E.D.F. 1995b. Schéma Directeur de Récupération des déchets flottants. Le Bouscat, rapport d'étude, $44 \mathrm{p}$.

E.D.F. 1995a. Barrage de Luzech (Lot). Etude des déchets flottants. Analyse qualitative et quantitative, provenances, modes de récupération et de traitements. Brive, rapport d'étude, n.p.

Gregory, S. V., Boyer, K. \& Gurnell, A. M. (dir.) 2003. The Ecology and Management of Wood in World Rivers. Bethesda, American Fisheries Society, 431 p.

Institution Interdépartementale pour l'Aménagement Hydraulique du bassin de l'Adour 1999. Récupération et traitement des déchets et corps flottants du bassin de l'Adour et du littoral. Schéma directeur. Toulouse, rapport d'étude, $47 \mathrm{p}$.

Institution Interdépartementale pour l'Aménagement Hydraulique du bassin de l'Adour 1998. Récupération et traitement des déchets flottants du bassin de l'Adour et du littoral : étude de définition. Toulouse, rapport d'étude, $87 \mathrm{p}$.

Le Lay, Y.-F. 2005. Y a-t-il une place pour le bois dans la rivière aménagée ?. Aestuaria, 7, 437458.

Le Lay, Y.-F. \& Piégay, H. 2007. Le bois mort dans les paysages fluviaux français : éléments pour une gestion renouvelée. L'Espace géographique, 1, 51-64

Le Lay, Y.-F., Piégay, H. \& Cossin M. 2006. Les enquêtes de perception paysagère à l'aide de photographies : choix méthodologiques et exemples en milieu fluvial. Septièmes Rencontres de Théo Quant à Besançon du 26 au 28 janvier 2005. http://thema.univfcomte.fr/theoq/pdf/2005/LeLay-theoquant05.pdf.

Ministère de l'Ecologie et du Développement Durable 2002. Petite hydroélectricité et environnement. Rapport $d u$ groupe de travail. Paris, MEDD, $95 \mathrm{p}$. http://www.ecologie.gouv.fr/IMG/pdf/rapport-petite-hydro.pdf.

Moulin B. 2005. Variabilité spatiale et temporelle du bois dans le réseau hydrographique de l'Isère à l'amont de Grenoble. Université Jean Monnet - Saint-Etienne, thèse de Doctorat, 450 p.

Moulin, B. 1999. La dynamique du bois mort à l'échelle de bassins versants : analyse prospective. Université Jean Monnet - Saint-Etienne, mémoire de D.E.A., 67 p.

Moulin, B. \& Grosprêtre, L. 2005. Etude des corps flottants transitant dans le Rhône et au droit des aménagements hydroélectriques. Lyon, rapport d'étude CNR-CNRS, 135 p. 
Moulin, B. \& Piégay, H. 2004. Characteristics and temporal variability of large woody debris trapped in a reservoir on the river Rhône (Rhône): implications for river basin management. River Research and Applications, 20, 79-97.

Piégay, H., Le Lay, Y.-F. \& Moulin, B. 2005. Les risques liés aux embâcles de bois dans les cours d'eau : état des connaissances et principes de gestion. In Vallauri, D., André, J., Dodelin, B., Eynard-Machet, R. \& Rambaud, D. (coord.), Bois mort et à cavités. Une clé pour des forêts vivantes. Paris, Lavoisier et Editions Tec \& Doc, 193-202.

Schéma Directeur d'Aménagement et de Gestion des Eaux Adour-Garonne 1996. http://www.eauadour-garonne.fr/sdage/default.html.

SMEAG / EPTB Garonne 2004. La vallée de la Garonne, un territoire transfrontalier. Gestion des déchets flottants. Montrejeau, rapport d'étude, $47 \mathrm{p}$. 


\section{Liste des tableaux}

Tableau 1. Deux approches du tri des déchets flottants.

\begin{tabular}{|l|l|l|}
\hline Avantages & \multicolumn{1}{|c|}{ Séparation du bois } & \multicolumn{1}{|c|}{ Séparation multiflux } \\
\hline Inconvénients & $\begin{array}{l}\text { - Pas de tri manuel. } \\
\text { - Coût modéré. } \\
\bullet \text { Main d'œuvre peu mobilisée. }\end{array}$ & $\begin{array}{l}\text { - Valorisation théorique optimale. } \\
\text { Traitement des déchets } \\
\text { toxiques. }\end{array}$ \\
\hline & $\begin{array}{l}\bullet \text { Présence de bois souillés. } \\
\text { - Valorisation du bois limitée. } \\
\text { - Médiocre séparation des toxiques. du tri manuel. } \\
\text { - Pas de traitement des produits } \\
\text { recyclables. }\end{array}$ & $\begin{array}{l}\text { - Coût élevé. } \\
\text { Main d'œuvre importante. } \\
\text { nécessaires. }\end{array}$ \\
\hline
\end{tabular}


Tableau 2. Les déchets flottants dans les filières locales de traitement.

\begin{tabular}{|c|c|c|c|}
\hline Filière & Type de bois & Avantages & Inconvénients \\
\hline Enfouissement & \multicolumn{3}{|c|}{ Interdiction légale } \\
\hline $\begin{array}{l}\text { Brûlage en plein } \\
\text { air }\end{array}$ & \multicolumn{3}{|c|}{ Interdiction légale } \\
\hline $\begin{array}{l}\text { Incinération } \\
\text { en U.I.O.M. }\end{array}$ & $\begin{array}{l}\text { Tous les bois } \\
\text { (après déferraillage et } \\
\text { broyage) }\end{array}$ & $\begin{array}{l}\text { Tri peu } \\
\text { contraignant }\end{array}$ & $\begin{array}{l}\text { - Broyeur puissant } \\
\text { - Coût élevé }\end{array}$ \\
\hline $\begin{array}{l}\text { Chaudière à } \\
\text { Alimentation } \\
\text { Automatique }\end{array}$ & Presque tous les bois & $\begin{array}{l}\text { Vente } \mathrm{du} \\
\text { combustible }\end{array}$ & $\begin{array}{l}\text { - Tri des déchets } \\
\text { - Broyage adéquat } \\
\text { - Stockage et séchage des } \\
\text { plaquettes }\end{array}$ \\
\hline Compostage & $\begin{array}{l}\text { Déchets } \\
\text { biodégradables }\end{array}$ & $\begin{array}{l}\text { Vente } \quad \mathrm{du} \\
\text { produit } \\
\text { fertilisant }\end{array}$ & $\begin{array}{l}\text { - Tri des déchets } \\
\text { - Broyage adéquat }\end{array}$ \\
\hline
\end{tabular}


Tableau 3. Les principaux combustibles du marché.

\begin{tabular}{|c|c|c|c|c|}
\hline $\begin{array}{l}\text { Origine } \\
\text { des sous- } \\
\text { produits }\end{array}$ & $\begin{array}{c}\text { Nature } \\
\text { des sous-produits }\end{array}$ & Conditionnement & & Combustible \\
\hline $\begin{array}{l}\text { Exploitations } \\
\text { forestières }\end{array}$ & $\begin{array}{l}\text { Rémanents } \\
\text { Bois sans valeur } \\
\text { Branches et cimes }\end{array}$ & Déchiquetage & & Plaquettes \\
\hline Scieries & $\begin{array}{l}\text { Ecorces } \\
\text { Sciures } \\
\text { Dosses et délignures } \\
\text { Diverses chutes } \\
\text { humides }\end{array}$ & $\begin{array}{l}\text { Utilisation directe } \\
\text { Broyage } \\
\text { Déchiquetage } \\
\text { Granulation (séchage } \\
\text { compression) }\end{array}$ & et & $\begin{array}{l}\text { Plaquettes } \\
\text { Ecorces } \\
\text { humides } \\
\text { Sciures } \\
\text { humides } \\
\text { Granulés }\end{array}$ \\
\hline $\begin{array}{l}\text { Menuiseries et } \\
\text { ameublement }\end{array}$ & $\begin{array}{lrr}\text { Sciures et } & \text { copeaux } \\
\text { secs } & & \\
\text { Diverses } & & \text { chutes } \\
\text { sèches } & & \\
\end{array}$ & $\begin{array}{l}\text { Utilisation directe } \\
\text { Déchiquetage } \\
\text { Granulation (séchage } \\
\text { compression) }\end{array}$ & & $\begin{array}{l}\text { Sciures et } \\
\text { copeaux secs } \\
\text { Plaquettes } \\
\text { Granulés }\end{array}$ \\
\hline $\begin{array}{l}\text { Centres de tri } \\
\text { des déchets } \\
\text { industriels } \\
\text { banals }\end{array}$ & $\begin{array}{l}\text { Bois flottants } \\
\text { Palettes et caisseries }\end{array}$ & $\begin{array}{l}\text { Broyage } \\
\text { Déferraillage }\end{array}$ & & Broyat \\
\hline
\end{tabular}




\section{Liste des figures}

Figure 1. Le dégrilleur du barrage de Saint-Egrève (cliché : E.D.F. Energie-Alpes, 2001).

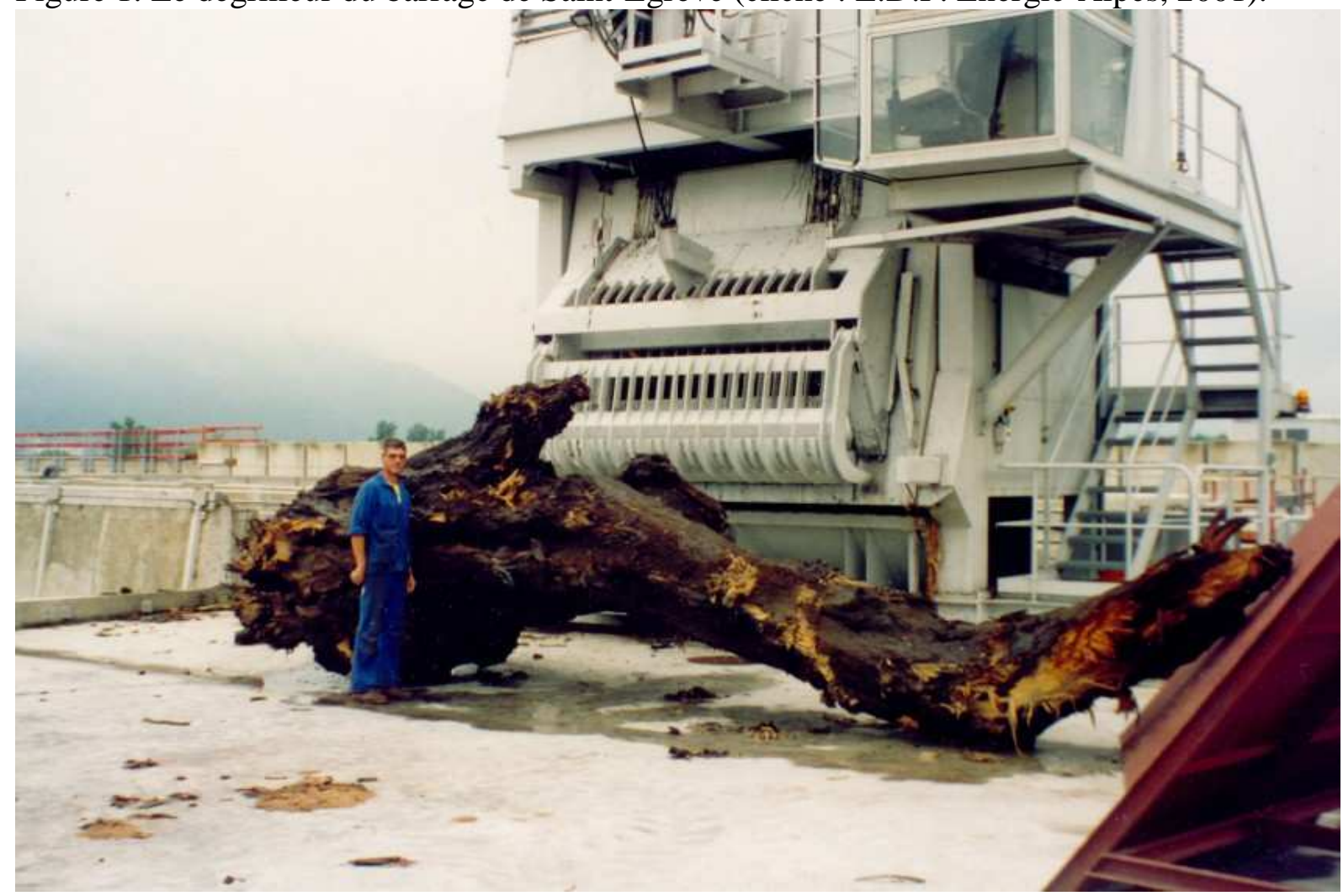


Figure 2. L'extraction des bois flottants au barrage de Génissiat (cliché : Moulin B., 1999).

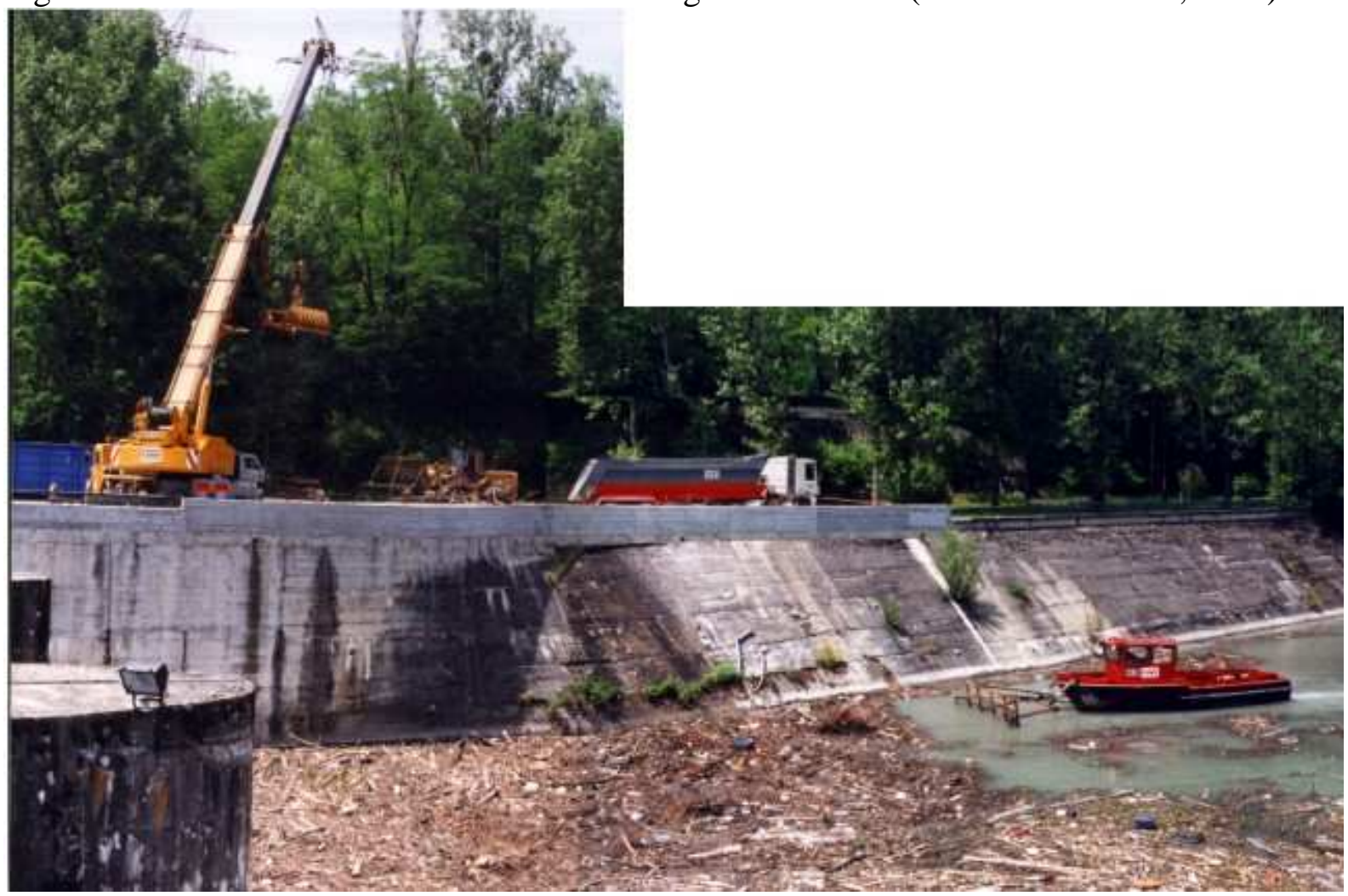


Figure 3. Les grandes étapes de la transformation du bois flottant.

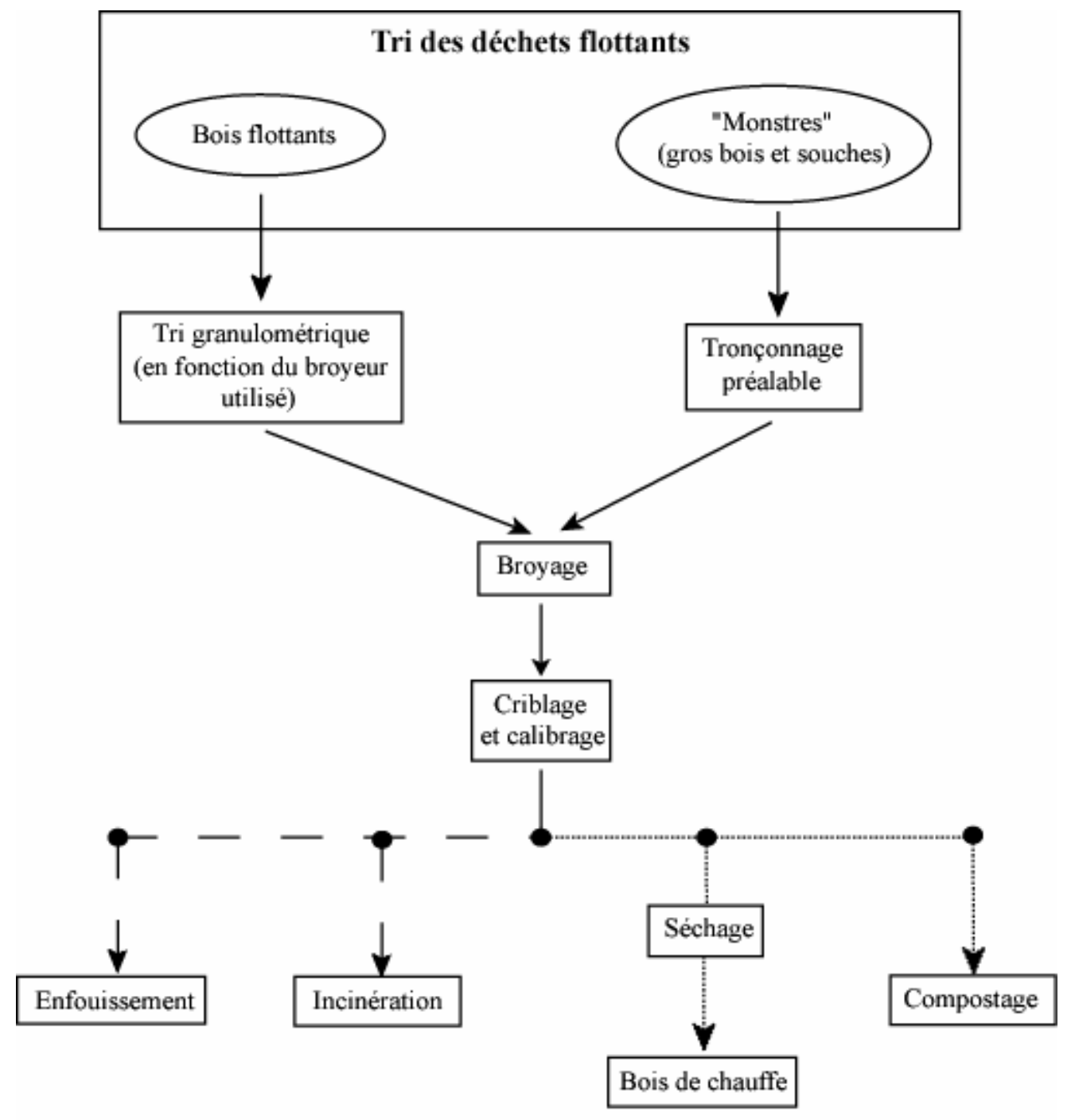

$\longrightarrow$ Elimination du bois
$\rightarrow$ Valorisation du bois 
Figure 4. Le broyage des bois flottants au barrage de Génissiat (cliché : Moulin B., 2005).

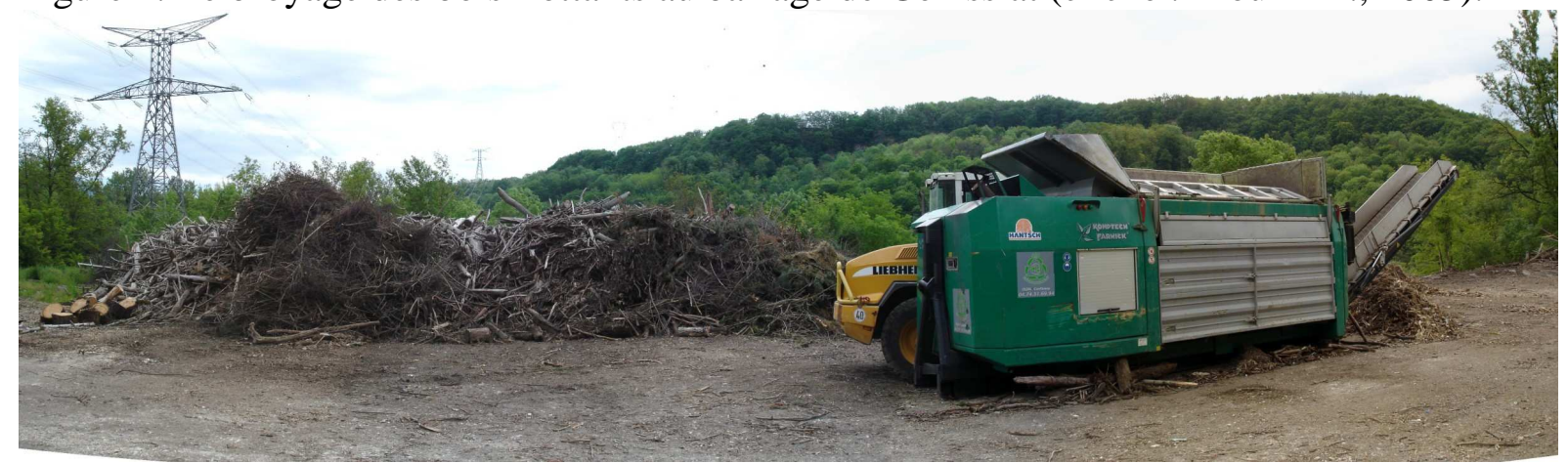


Figure 5. La filière de recyclage du bois en Isère.

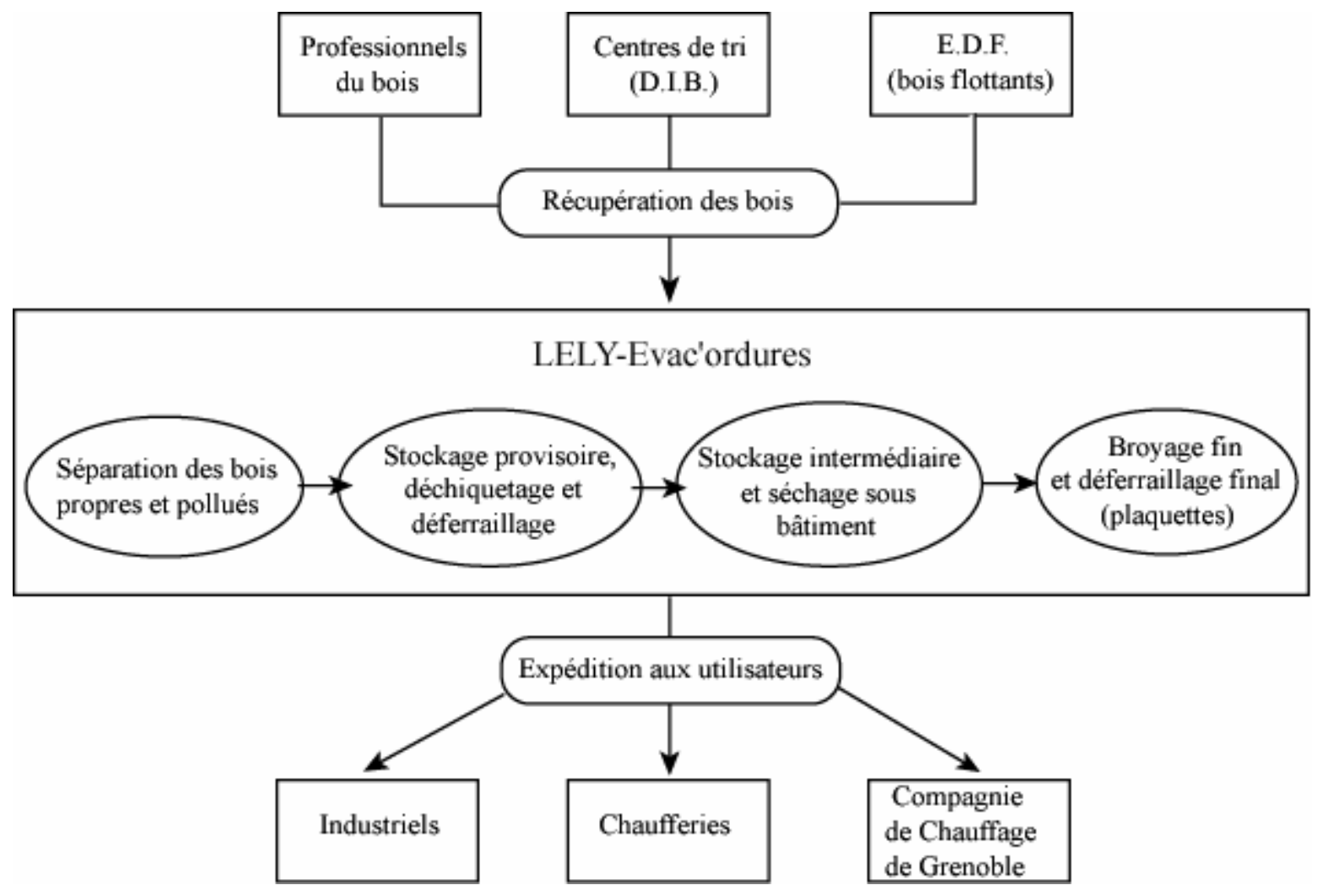

